Draft VERSion MARCh 8, 2018

Typeset using $\mathrm{LAT}_{\mathrm{E}} \mathrm{X}$ twocolumn style in AASTeX61

\title{
A NEW WINDOW INTO ESCAPING EXOPLANET ATMOSPHERES: 10830 Å LINE OF HELIUM
}

\author{
Antonija OKlopČić ${ }^{1}$ And Christopher M. Hirata ${ }^{2}$
}

\author{
${ }^{1}$ Institute for Theory and Computation, Harvard-Smithsonian Center for Astrophysics \\ 60 Garden Street, MS-51 \\ Cambridge, Massachusetts 02138, USA \\ ${ }^{2}$ Center for Cosmology and Astroparticle Physics, Ohio State University \\ 191 West Woodruff Avenue \\ Columbus, Ohio 43210, USA
}

\section{ABSTRACT}

Observational evidence for escaping exoplanet atmospheres has been obtained for a few exoplanets to date. It comes from strong transit signals detected in the ultraviolet, most notably in the wings of the hydrogen Lyman- $\alpha$ (Ly $\alpha$ ) line. However, the core of the Ly $\alpha$ line is often heavily affected by interstellar absorption and geocoronal emission, limiting the information about the atmosphere that can be extracted from that part of the spectrum. Transit observations in atomic lines that are (a) sensitive enough to trace the rarefied gas in the planetary wind and (b) do not suffer from significant extinction by the interstellar medium could enable more detailed observations, and thus provide better constraints on theoretical models of escaping atmospheres. The absorption line of a metastable state of helium at $10830 \AA$ could satisfy both of these conditions for some exoplanets. We develop a simple $1 \mathrm{D}$ model of escaping planetary atmospheres containing hydrogen and helium. We use it to calculate the density profile of helium in the $2^{3} \mathrm{~S}$ metastable excited state and the expected in-transit absorption at $10830 \AA$ for two exoplanets known to have escaping atmospheres. Our results indicate that exoplanets similar to GJ 436b and HD 209458b should exhibit enhanced transit depths at $10830 \AA$, with $\sim 8 \%$ and $\sim 2 \%$ excess absorption in the line core, respectively.

Keywords: atomic processes — radiative transfer — planets and satellites: atmospheres — planets and satellites: gaseous planets 


\section{INTRODUCTION}

Close-in exoplanets give us a new insight into the mechanisms of atmospheric escape and mass loss. In highly irradiated planets, atmospheric escape can be very efficient and act collectively on the atmosphere as a fluid (e.g. Owen \& Jackson 2012), instead of on a particle-by-particle basis. This hydrodynamic escape may be important for the planetary evolution, especially in low-mass planets that are more vulnerable to photoevaporation compared to massive planets with deep gravitational wells. This process has been proposed as an explanation for the observed paucity of short-period subJupiter planets and the bimodal distribution of planet radii (Owen \& Wu 2013; Lundkvist et al. 2016; Fulton et al. 2017). Improving our knowledge of how hydrodynamic escape works and how it affects a broad range of atmospheres is therefore necessary for better understanding the demographics of planetary systems and their evolution.

Observational evidence for atmospheric escape has been obtained for a handful of exoplanets to date in the form of a strong absorption signal detected in the wings of the hydrogen Ly $\alpha$ line, but also in some UV lines of metals (Vidal-Madjar et al. 2003, 2004; Lecavelier Des Etangs et al. 2010; Linsky et al. 2010; Fossati et al. 2010; Kulow et al. 2014). The first observations of this kind were obtained for a transiting hot Jupiter HD 209458b by Vidal-Madjar et al. (2003). Strong absorption in the wings of the Ly $\alpha$ line resulted in transit depth about an order of magnitude greater than the optical transit, suggesting that the observed cloud of hydrogen extends far away from the planet. Even greater transit depths in the wings of Ly $\alpha$ have been reported for a warm Neptune GJ 436b (Kulow et al. 2014; Ehrenreich et al. 2015; Lavie et al. 2017).

Several groups have developed theoretical models of escaping atmospheres (e.g. Lammer et al. 2003; Yelle 2004; García Muñoz 2007; Murray-Clay, Chiang \& Murray 2009; Koskinen et al. 2010; Bourrier \& Lecavelier des Etangs 2013; Tripathi et al. 2015; Salz et al. 2016; Carroll-Nellenback et al. 2017). The methodology and the complexity varies greatly between these models, and hence their predictions, such as the expected mass loss rate, can differ by orders of magnitude. More detailed observations are required to place more stringent constraints on theoretical models.

Ly $\alpha$ observations have been immensely valuable for providing evidence of atmospheric escape. However, there are inherent limitations of using this line. Ly $\alpha$ suffers from extinction by the interstellar medium and contamination from geocoronal emission, rendering the signal from the Ly $\alpha$ line core - and the valuable informa-

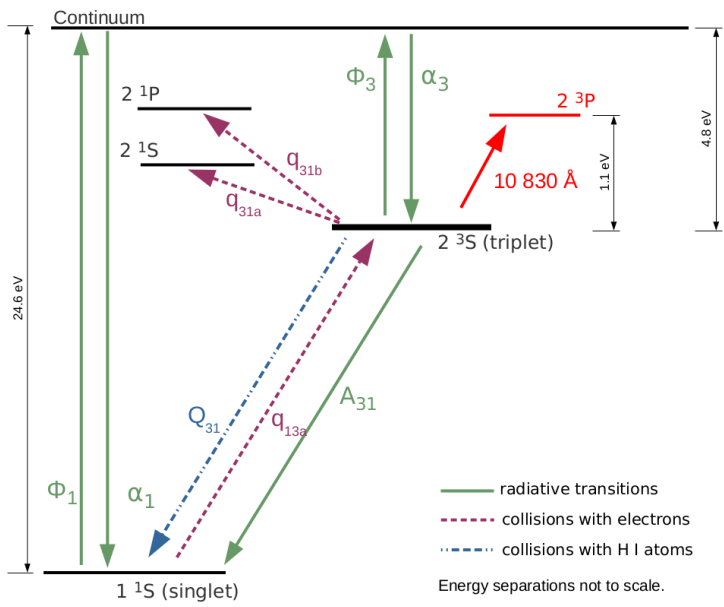

Figure 1. Structure of the helium atom, indicating the radiative and collisional transitions included in our analysis. The transition shown in red depicts the $10830 \AA$ absorption line.

tion content it might carry-irretrievable (e.g. Ehrenreich et al. 2015).

Here, we investigate the possibility of probing the escaping atmospheres of exoplanets with the absorption line of helium at $10830 \AA$. This line may provide a new wavelength window for studying the hydrodynamic escape and atmospheric mass loss. Its main advantages over the UV lines include weaker interstellar absorption ${ }^{1}$ and the possibility of ground-based observations.

\section{HELIUM METASTABLE STATE AND THE $10830 \AA$ LINE}

The helium atom can exist in two configurations based on the relative orientation of its electrons' spin, singlet (anti-parallel) and triplet (parallel). The lowest-lying triplet level $\left(2^{3} \mathrm{~S}\right)$ is almost decoupled from the singlet ground state $\left(1^{1} \mathrm{~S}\right)$ because radiative transitions between them are strongly suppressed. Due to relativistic and finite-wavelength corrections to the magnetic dipole transition formula, the $2^{3} \mathrm{~S}$ triplet helium can radiatively decay into the singlet ground state with an exceptionally long lifetime of $2.2 \mathrm{hr}$ (Drake 1971).

The $2^{3} \mathrm{~S}$ state can be populated by recombination ${ }^{2}$ or by collisional excitation from the ground state (see Figure 1). Depopulation of this state progresses slowly making it metastable, and hence a promising origin of

\footnotetext{
1 Indriolo et al. (2009) measured the column density of metastable helium through diffuse interstellar clouds and obtained an upper limit of $N \lesssim 10^{9} \mathrm{~cm}^{-2}$, which is roughly three orders of magnitude lower than our prediction for escaping exoplanet atmospheres (see Figure 3, bottom panel).

2 Around $75 \%$ of helium recombinations result in the triplet configuration (Osterbrock \& Ferland 2006).
} 
absorption lines. The resonance scattering from the metastable state to the $2^{3} P$ state produces an absorption line at $10830 \AA$. This transition consists of three lines, two of which —at $10830.34 \AA$ and $10830.25 \AA$-are practically indistinguishable, whereas the third component is at $10829.09 \AA$.

The helium $10830 \AA$ line has been used to probe the dynamics of stellar winds (e.g. Dupree, Sasselov \& Lester 1992; Edwards et al. 2003) and outflows from active galactic nuclei (e.g. Leighly, Dietrich \& Barber 2011), as well as the structure of the solar chromosphere and transition region (Avrett 1994; Andretta \& Jones 1997; Mauas et al. 2005). Even though this line has been identified as one of the most promising spectral signatures of exoplanet atmospheres (Seager \& Sasselov 2000), no firm detections have been reported thus far (see, e.g., Moutou et al. 2003).

Most previous models of escaping atmospheres have focused on hydrogen or tracked helium only in terms of neutrals and ions, without modeling the metastable state. Turner et al. (2016) used CLOUDY to compile a list of $\sim 60$ potentially interesting absorption lines for probing upper exoplanet atmospheres, including the $10830 \AA$ Å line.

\section{METHODS AND RESULTS}

\subsection{Isothermal Parker Wind}

Our model is based on the assumption that upper layers of a hydrodynamically escaping atmosphere can be described by an isothermal Parker wind driven by gas pressure (Parker 1958; Watson 1981). Winds in which heating and cooling are due to radiative processes are close to being isothermal (Lamers \& Cassinelli 1999). If the radiative heating/cooling processes operate on short timescales compared to the dynamical time of the system, the gas can self-regulate at a constant temperature.

A time-independent and spherically symmetric wind has a constant mass-loss rate determined by the equation of mass conservation:

$$
\dot{M}=4 \pi r^{2} \rho(r) v(r) .
$$

For an isothermal wind, the energy equation is

$$
T(r)=T_{0},
$$

whereas the momentum equation is given by

$$
v \frac{d v}{d r}+\frac{1}{\rho} \frac{d p}{d r}+\frac{G M_{p l}}{r^{2}}=0 .
$$

We ignore the gravitational influence of the star and the Coriolis force. The momentum equation has a singularity at the so-called critical point, which in our case coincides with the sonic point, where the wind velocity equals the isothermal speed of sound

$$
v_{s}=\sqrt{\frac{k T_{0}}{\mu m_{H}}},
$$

where $\mu m_{H}$ is the mean molecular weight. We assume that gas is made up of $90 \%$ (by number) atomic hydrogen and $10 \%$ helium $^{3}$. The radius of the sonic point is

$$
r_{s}=\frac{G M_{p l}}{2 v_{s}^{2}} .
$$

The velocity profile of the isothermal wind is given by (Lamers \& Cassinelli 1999):

$$
\frac{v(r)}{v_{s}} \exp \left[-\frac{v^{2}(r)}{2 v_{s}^{2}}\right]=\left(\frac{r_{s}}{r}\right)^{2} \exp \left(-\frac{2 r_{s}}{r}+\frac{3}{2}\right) .
$$

Using the mass continuity equation (Equation 1), we get the equation for the density profile:

$$
\frac{\rho(r)}{\rho_{s}}=\exp \left[\frac{2 r_{s}}{r}-\frac{3}{2}-\frac{v^{2}(r)}{2 v_{s}^{2}}\right] .
$$

We use these expressions to set the density and velocity structure of the planetary wind. The free parameters in our model are the planet mass, radius, wind temperature and mass loss rate. We choose these parameters so that they match the properties of two well-studied exoplanets known to have escaping atmospheres: GJ 436b (Butler et al. 2004), a Neptune-sized planet orbiting an M-type star, and HD 209458b (Henry et al. 2000; Charbonneau et al. 2000), a hot Jupiter around a Sun-like star. Our model cannot predict the wind parameters such as the temperature and mass-loss rate, so we must assume their values. Our choice of $T_{0}=5 \times 10^{3} \mathrm{~K}$ and $\dot{M}=2 \times 10^{10} \mathrm{~g} \mathrm{~s}^{-1}$ for GJ $436 \mathrm{~b}$, and $T_{0}=9 \times 10^{3} \mathrm{~K}$ and $\dot{M}=8 \times 10^{10} \mathrm{~g} \mathrm{~s}^{-1}$ for HD 209458b, is guided by the results of Salz et al. (2016), who used a hydrodynamics code coupled with CLOUDY (Salz et al. 2015) to model atmospheric heating and wind launching for a number of known exoplanets.

In Figure 2, we show our velocity and density profiles for both planets. Despite the simplicity of the isothermal model, the obtained atmospheric structure is very similar to the results of Salz et al. (2016) simulations, shown for comparison.

\footnotetext{
3 One of the planets whose escaping atmosphere we model (GJ 436b) has been suggested to have a helium-rich atmosphere (Hu, Seager \& Yung 2015). This could have important implications for $10830 \AA$ absorption; however, we leave the investigation of atmospheres with different compositions for future work.
} 


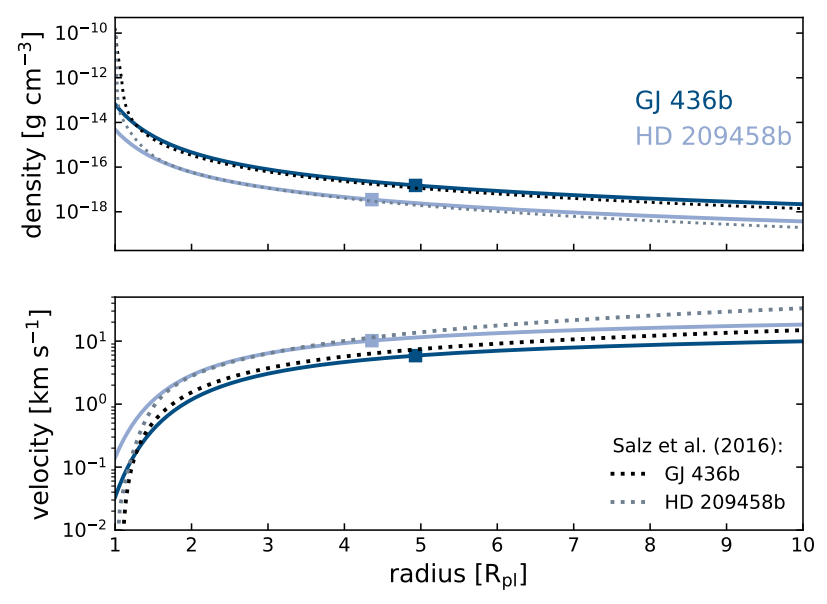

Figure 2. Density and velocity profiles of the isothermal Parker wind for GJ 436b and HD 209458b. Squares mark the sonic point. Dotted lines show the corresponding profiles obtained by Salz et al. (2016).

\subsection{Steady-state Hydrogen Distribution}

First, we calculate the radial density profile for hydrogen atoms and ions. The steady-state advection and recombination/ionization balance can be written as

$$
\frac{1}{r^{2}} \frac{\partial}{\partial r}\left(r^{2} n_{\mathrm{H}^{0}} v\right)=n_{\mathrm{H}^{+}} n_{e} \alpha_{r e c}-n_{\mathrm{H}^{0}} \Phi^{\prime}
$$

where $n_{\mathrm{H}^{0}}, n_{\mathrm{H}^{+}}$, and $n_{e}$ denote the number density of neutral hydrogen, ionized hydrogen, and electrons, respectively, $v$ is the (radial) velocity of the outflow, $\alpha_{r e c}=2.59 \times 10^{-13}\left(T_{0} / 10^{4}\right)^{-0.7} \mathrm{~cm}^{3} \mathrm{~s}^{-1}$ is the case-B hydrogen recombination rate at $T_{0}$ (Osterbrock \& Ferland 2006; Tripathi et al. 2015), and $\Phi^{\prime}$ is the hydrogen photoionization rate calculated as

$$
\Phi^{\prime}=\int_{\nu_{0}}^{\infty} \frac{F_{\nu}}{h \nu} a_{\nu} e^{-\tau} d \nu \approx e^{-\tau_{0}} \int_{\nu_{0}}^{\infty} \frac{F_{\nu}}{h \nu} a_{\nu} d \nu \equiv e^{-\tau_{0}} \Phi
$$

where $\nu_{0}$ is the frequency corresponding to $13.6 \mathrm{eV}$ photons. The hydrogen photoionization cross section $a_{\nu}$ is given by (Osterbrock \& Ferland 2006):

$$
a_{\nu}=6.3 \times 10^{-18} \frac{\exp \left(4-\frac{4 \tan ^{-1} \epsilon}{\epsilon}\right)}{1-\exp (-2 \pi / \epsilon)}\left(\frac{\nu_{0}}{\nu}\right)^{4}\left[\mathrm{~cm}^{2}\right],
$$

where $\epsilon=\sqrt{\nu / \nu_{0}-1}$. We calculate $\tau_{0}$ as

$$
\tau_{0}=a_{0} \int_{r}^{\infty} n_{\mathrm{H}^{0}} d r=\frac{0.9 a_{0}}{1.3 m_{H}} \int_{r}^{\infty}\left(1-f_{\text {ion }}\right) \rho(r) d r,
$$

where $\frac{0.9 \rho}{1.3 m_{\mathrm{H}}}$ is the number density of hydrogen nuclei, $f_{\text {ion }}=n_{\mathrm{H}^{+}} /\left(n_{\mathrm{H}^{0}}+n_{\mathrm{H}^{+}}\right)$is the ionized fraction of hydrogen (we assume $n_{e}=n_{\mathrm{H}^{+}}$), and $a_{0}$ is the flux-averaged photoionization cross section given by

$$
a_{0}=\frac{\int_{\nu_{0}}^{\infty} F(\nu) a_{\nu} d \nu}{\int_{\nu_{0}}^{\infty} F(\nu) d \nu}
$$

where $F(\nu)$ is the stellar flux in units of photons $/\left(\mathrm{cm}^{2}\right.$ $\mathrm{s} \mathrm{Hz})$. Here we ignore the effects of radiative processes involving helium: optical depth in Equation 11 does not account for helium absorption nor the hydrogen-ionizing photons produced by helium recombination.

$F_{\nu}$ in Equation 9 denotes the stellar flux density. For GJ 436, we use the MUSCLES survey data, version 2.1 (France et al. 2016; Youngblood et al. 2016; Loyd et al. 2016). For HD 209458, a G0-type star, we use the SORCE solar spectral irradiance data from the LASP Interactive Solar Irradiance Datacenter ${ }^{4}$. Because HD 209458 is an inactive star (Czesla et al. 2017, and references therein), we use the solar spectrum data recorded during a solar minimum ${ }^{5}$. To fill in a gap in the data in the wavelength range $\sim 400-1150 \AA$, we use the scaling relations between the Ly $\alpha$ flux and fluxes in EUV bands from Linsky et al. (2014).

Using the continuity equation (Equation 1), Equation 8 can be written as

$$
\frac{\partial f_{\text {ion }}}{\partial r}=\frac{1-f_{\text {ion }}}{v} \Phi e^{-\tau_{0}}-\frac{0.9 \rho}{1.3 m_{\mathrm{H}} v} f_{\text {ion }}^{2} \alpha_{r e c} .
$$

The calculated fractions of neutral and ionized hydrogen as functions of altitude are shown in Figure 3.

\subsection{Steady-state Helium Distribution}

Similarly to our treatment of hydrogen, we can derive steady-state equations for the radial distribution of helium atoms:

$$
\begin{aligned}
v \frac{\partial f_{1}}{\partial r} & =\left(1-f_{1}-f_{3}\right) n_{e} \alpha_{1}+f_{3} A_{31}-f_{1} \Phi_{1} e^{-\tau_{1}} \\
& -f_{1} n_{e} q_{13 a}+f_{3} n_{e} q_{31 a}+f_{3} n_{e} q_{31 b}+f_{3} n_{\mathrm{H}^{0}} Q_{31}, \quad(14) \\
v \frac{\partial f_{3}}{\partial r} & =\left(1-f_{1}-f_{3}\right) n_{e} \alpha_{3}-f_{3} A_{31}-f_{3} \Phi_{3} e^{-\tau_{3}}+f_{1} n_{e} q_{13 a} \\
& -f_{3} n_{e} q_{31 a}-f_{3} n_{e} q_{31 b}-f_{3} n_{\mathrm{H}^{0}} Q_{31} .
\end{aligned}
$$

$f_{1}$ and $f_{3}$ mark the fractions of helium in the (neutral) singlet and triplet state, respectively. $\alpha$ and $\Phi$ are the recombination and photoionization rate coefficients. We use the photoionization cross sections for the metastable triplet state from Norcross (1971). For the singlet state,

\footnotetext{
4 http://lasp.colorado.edu/lisird/

5 The population of the helium triplet state is very sensitive to the stellar UV flux. Using a solar spectrum from a more active period produces a stronger $10830 \AA$ absorption feature for HD 209458b.
} 
we use the photoionization cross section from Brown (1971). Recombination rates are from Osterbrock \& Ferland (2006). For simplicity, we assume that the number density of electrons is equal to the number density of ionized hydrogen atoms (i.e. we ignore the electrons produced by helium ionization, which can increase $n_{e}$ by up to $\sim 10 \%$ ). Optical depths $\tau_{1}$ and $\tau_{3}$ are calculated using flux-averaged cross sections. For $\tau_{1}$, we take into account both helium and hydrogen absorption, and the threshold frequency in Equation 12 now corresponds to $24.6 \mathrm{eV}$. For $\tau_{3}$, we integrate over frequencies between the $2^{3} \mathrm{~S}$ ionization threshold $(4.8 \mathrm{eV})$ and the hydrogenionization threshold.

Transitions between singlet and triplet levels due to collisions with free electrons are described by coefficients

$q_{i j}=2.1 \times 10^{-8} \sqrt{\frac{13.6 \mathrm{eV}}{k T}} \exp \left(-\frac{E_{i j}}{k T}\right) \frac{\Upsilon_{i j}}{\omega_{i}}\left[\mathrm{~cm}^{3} \mathrm{~s}^{-1}\right]$,

where $\omega_{i}$ is the statistical weight of the initial level and $\Upsilon_{i j}$ is the effective collision strength from Bray et al. (2000). The values obtained are $q_{13 a}=4.5 \times 10^{-20} \mathrm{~cm}^{3}$ $\mathrm{s}^{-1}, q_{31 a}=2.6 \times 10^{-8} \mathrm{~cm}^{3} \mathrm{~s}^{-1}$, and $q_{31 b}=4.0 \times$ $10^{-9} \mathrm{~cm}^{3} \mathrm{~s}^{-1}$. The metastable triplet level can also be depopulated by collisions with neutral hydrogen atoms via associative ionization

$$
\mathrm{He}\left(2^{3} \mathrm{~S}\right)+\mathrm{H} \rightarrow \mathrm{HeH}^{+}+\mathrm{e},
$$

and Penning ionization

$$
\mathrm{He}\left(2^{3} \mathrm{~S}\right)+\mathrm{H} \rightarrow \mathrm{He}+\mathrm{H}^{+}+\mathrm{e} .
$$

The combined rate coefficient for these processes is $Q_{31} \sim 5 \times 10^{-10} \mathrm{~cm}^{3} \mathrm{~s}^{-1}$ (Roberge \& Dalgarno 1982). The radiative transition rate between the metastable and ground state is given by $A_{31}=1.272 \times 10^{-4} \mathrm{~s}^{-1}$ (Drake 1971), which is one of the slowest rates in our reaction network.

Figure 1 shows a schematic view of all the radiative and collisional transitions included in our calculation. The radiative transition between the metastable state and the $2^{3} \mathrm{P}$ state - which is the origin of the $10830 \AA$ line - is not included in the reaction network because this process does not significantly depopulate the metastable state (i.e. it conserves the triplet configuration because an atom in the $2^{3} \mathrm{P}$ state just decays back into the $2^{3} \mathrm{~S}$ state). We also neglect collisional ionization of helium, which is reasonable considering the assumed wind temperatures. We ignore direct transitions from the metastable to the ground state due to electron collisions because they are less probable than collisional transitions to the excited singlet states (Osterbrock \& Ferland 2006).
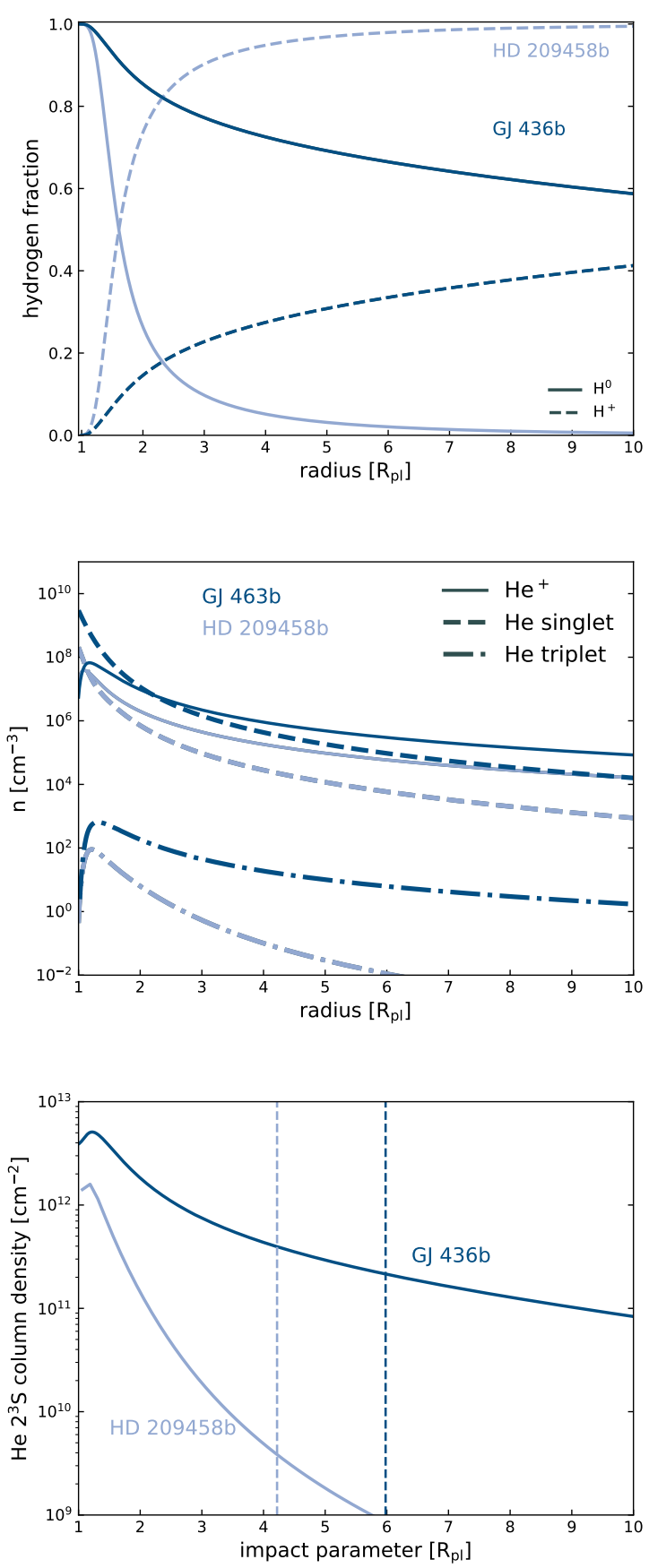

Figure 3. Top: density profiles of neutral and ionized hydrogen. Middle: density profile of the ionized, ground state (singlet) and metastable (triplet) helium. Bottom: column density of metastable helium as a function of distance from the planet. Dashed lines mark the Roche-lobe radii.

The calculated density profiles of the singlet and triplet helium are shown in the middle panel of Figure 3.

\subsection{Helium Line Absorption Profile in Transit}


Finally, we calculate the expected absorption of stellar light at $10830 \AA$ due to the presence of an escaping planetary atmosphere. For absorption along a line of sight with an impact parameter $b$, the optical depth is given by (see, e.g. Koskinen et al. 2010)

$$
\tau_{\nu}(b)=2 \int_{b}^{\infty} \frac{n_{3}(r) \sigma_{0} \Phi(\Delta \nu) r d r}{\sqrt{r^{2}-b^{2}}},
$$

where $n_{3}$ is the number density of the metastable triplet helium. The absorption cross section is

$$
\sigma_{0}=\frac{\pi e^{2}}{m_{e} c} f
$$

where $e$ and $m_{e}$ are the electron charge and mass, respectively, $c$ is the speed of light, and $f$ is the oscillator strength for the $10830 \AA$ transition (taken from the NIST Atomic Spectra Database ${ }^{6}$ for all three components of the line triplet). $\Phi(\Delta \nu)$ is the Voigt line profile ${ }^{7}$ with the half-width at half-maximum (HWHM) of the Gaussian part

$$
\alpha=\sqrt{\frac{2 \ln 2 k T_{0}}{m_{\mathrm{He}}}} \frac{\nu_{0}}{c},
$$

and the HWHM of the Lorentzian part $\gamma=A_{10830} / 4 \pi$, with the $A_{10830}=1.0216 \times 10^{7} \mathrm{~s}^{-1}$ value from NIST. The frequency offset from the line center $\Delta \nu=(\nu-$ $\left.\nu_{0}\right)-\frac{\nu_{0}}{c} v_{L O S}$ takes into account the line-of-sight component of the radial outflow velocity $v_{L O S}$ from our Parker wind velocity solution (see, e.g. Villarreal D'Angelo et al. 2014).

To compute the expected absorption line profile, we integrate $\tau_{\nu}(b)$ over the impact parameter from the planetary radius to the stellar radius, first by taking into account only the gas that is located within the planetary Roche lobe. We use the Roche-lobe height values from Salz et al. (2016), $5.98 \mathrm{R}_{\mathrm{pl}}$ for GJ $436 \mathrm{~b}$ and $4.22 \mathrm{R}_{\mathrm{pl}}$ for HD 209458b. The assumed spherical symmetry of the outflow is a reasonable approximation until the Roche radius. Beyond that, the wind can contribute to the absorption, but it may experience significant deviations from radial trajectories that can affect the line profile. To illustrate how much additional absorption could be caused by gas at larger radii, we also calculate the line profiles by taking into account gas at all radii.

Our main result, shown in Figure 4, is the in-transit absorption calculated for planets with GJ 436b-like and HD 209458b-like properties, transiting across the cen-

\footnotetext{
6 https://www.nist.gov/pml/atomic-spectra-database

7 https://scipython.com/book/chapter-8-scipy/examples/ the-voigt-profile/ (Hill 2016)
}

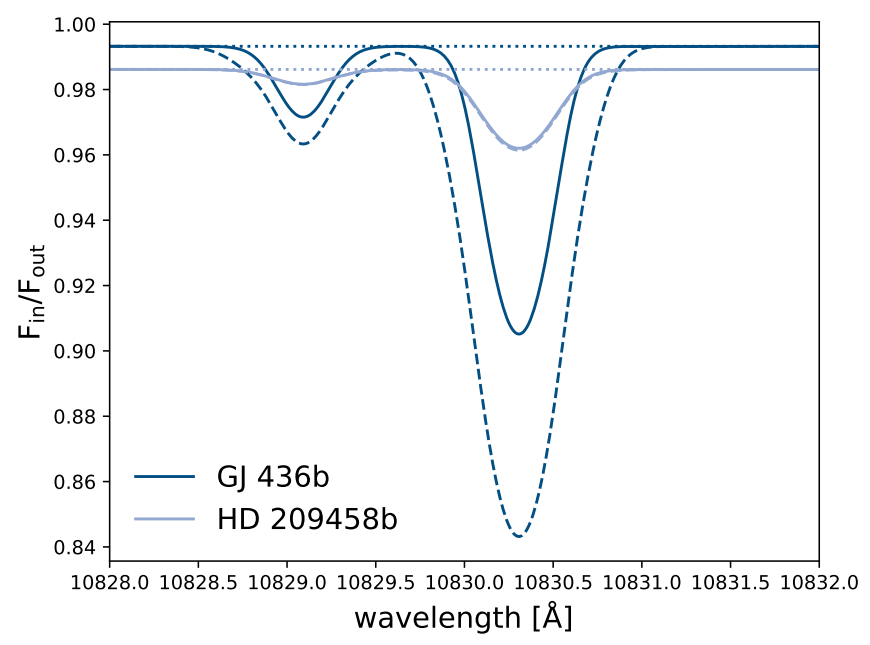

Figure 4. Calculated transmission spectra for planets with properties similar to GJ 436b and HD 209458b, transiting across the center of the stellar disk. Solid lines show the absorption caused by the metastable helium within the planetary Roche lobe, whereas the dashed lines show the absorption caused by gas at all altitudes. Dotted lines mark the optical transit depths.

ter of their host $\operatorname{star}^{8}$. The equivalent width (EW) of the helium absorption feature (i.e. excess absorption in addition to the planet's optical transit depth) for GJ $436 \mathrm{~b}$ is $0.047 \AA$ for gas up to the Roche radius (solid line) and $0.105 \AA$ for gas at all radii (dashed line). For HD 209458b, EW $=0.014 \AA$. The dotted lines in Figure 4 represent the optical transit depths, equal to $R_{\mathrm{pl}}^{2} / R_{*}^{2} \approx 0.69 \%$ and $1.4 \%$, for GJ $436 \mathrm{~b}$ and HD 209458b, respectively.

We validate the procedure described in this section by calculating the in-transit absorption in the hydrogen Ly $\alpha$ line and comparing our results to observational and theoretical studies from the literature, as shown in Appendix A.

\section{DISCUSSION AND CONCLUSIONS}

We develop a 1D model of escaping exoplanet atmospheres and use it to predict in-transit absorption signal in the $10830 \AA$ line of helium. If observed, the full absorption line profile at $10830 \AA$ would provide valuable information about the wind structure, including the base of the wind, which is currently lacking. This information would be complementary to that obtained in the extended wings of the Ly $\alpha$ line (because they probe dif-

\footnotetext{
${ }^{8}$ GJ 436b does not transit across the center of its star, but is at a projected distance of $\sim 0.85 R_{*}$. Consequently, a large fraction of the escaping gas does not transit the stellar disk and hence does not contribute to absorption.
} 
ferent parts of the outflow), and would therefore help constrain theoretical models of escaping exoplanet atmospheres.

Seager \& Sasselov (2000) predicted a strong absorption at $10830 \AA$ for HD 209458b. Their study was focused on the stable, lower atmosphere of the planet; however, they state that extremely large transit signals could be expected from an extended exosphere. Moutou et al. (2003) measured the transit of HD 209458b at $10830 \AA$ and placed an upper limit of $0.5 \%$ transit depth for a $3 \AA \AA$-wide line. Our model predicts a spectral feature of comparable EW, but much deeper and narrower. Given the significant differences in the line morphology and the fact that EWs differ by $\lesssim 6 \%$, a detailed analysis would be required to determine whether our prediction is consistent with Moutou et al. (2003) observation. However, this level of discrepancy could also be caused by the uncertainties in the input parameters we assume. Turner et al. (2016) model the upper exoplanet atmosphere as a static slab of uniform-density $\left(n \sim 10^{9} \mathrm{~cm}^{-3}\right)$ gas. They predict transit depths for dozens of spectral lines, including the $10830 \AA$ line, for which they obtain a transit depth of $\sim 0.3 \%$ over $\mathrm{a} \sim 50 \AA$-wide bin (J. Turner, priv. comm.). That is about an order of magnitude higher EW than our result for HD 209458b, which could be explained by differences in model geometry. They assume that high-density gas covers $\sim 40 \%$ of the stellar disk in projection, whereas our density profile drops below $10^{9} \mathrm{~cm}^{-3}$ at $\sim 1.5 \mathrm{R}_{\mathrm{pl}}$, occulting only $\sim 3 \%$ of the stellar disk.

Our results indicate that exoplanets like GJ 436b and HD 209458b should show enhanced absorption at $10830 \AA$ due to the presence of helium in the metastable state in their planetary wind. Based on our calculations, a GJ 436b-like planet is a more promising candidate for detecting the $10830 \AA$ line than a planet like HD 209458b. This is caused by the combined effect of (a) the fact that escaping atmospheres in planets with low gravitational potential tend to be denser at high altitudes, (b) different levels of helium ionization, (c) more favorable flux ratio of radiation responsible for populating versus depopulating the metastable state, and (d) differences in the hydrogen-ionizing radiation, which controls the hydrogen ionization fraction and electron density and, consequently, the relative contribution of different collisional depopulation mechanisms of the metastable state. We leave a more detailed investigation of how various stellar and planetary properties affect the expected absorption at $10830 \AA$ for future work.

In deriving our wind model, we make a number of simplifying assumptions. We perform our calculation in 1D and assume the outflow is spherically symmetric. According to the results of Khodachenko et al. (2015), this approximation is valid at altitudes up to a few planetary radii, where most of our signal comes from. 3D simulations find dayside to nightside differences in wind properties, and a 'cometary' tail of wind material at large distances behind the planet (e.g. Tripathi et al. 2015; Christie, Arras \& Li 2016; Schneiter et al. 2016; CarrollNellenback et al. 2017), which our 1D model cannot reproduce. We do not model the interaction between the escaping material and the stellar wind (e.g. Bourrier et al. 2016). Simulations of atmospheric heating and wind launching suggest that planetary winds are not strictly isothermal, which is another assumption that we make. Although this assumption does not greatly affect the wind density and velocity structure (Figure 2), it might have more subtle effects on the spectral line profile. A major limitation of our model is that we have to assume values for the wind temperature and mass-loss rate, and cannot predict them in a self-consistent way.

The main advantage of our model is that it is computationally less expensive than hydrodynamic simulations. This will allow us to explore a wide range of planetary parameters and stellar spectral types in our future work, in order to identify what part of the parameter space is most promising for producing strong absorption signals in the $10830 \AA$ line. Once we identify the best candidates, more computational resources can be invested into performing detailed studies of these systems, using 2D or 3D simulations with more physically motivated treatment of wind launching.

We thank the anonymous referee for providing very helpful comments. We thank David Charbonneau, Andrea Dupree, and Jessica Spake for insightful conversations. AO acknowledges support from an ITC Fellowship. CMH is supported by NASA, NSF, the U.S. Department of Energy, the David \& Lucile Packard Foundation, and the Simons Foundation.

Software: matplotlib (Hunter et al. 2007), numpy (Van Der Walt et al. 2011), scipy (Jones et al. 2001)

\section{APPENDIX}

\section{A. LY $\alpha$ ABSORPTION}

In addition to the helium $10830 \AA$ line, we can use our model to calculate the absorption in the hydrogen Ly $\alpha$ line. Following the procedure described in subsection 3.4, we calculate the optical depth to neutral hydrogen (protium and 

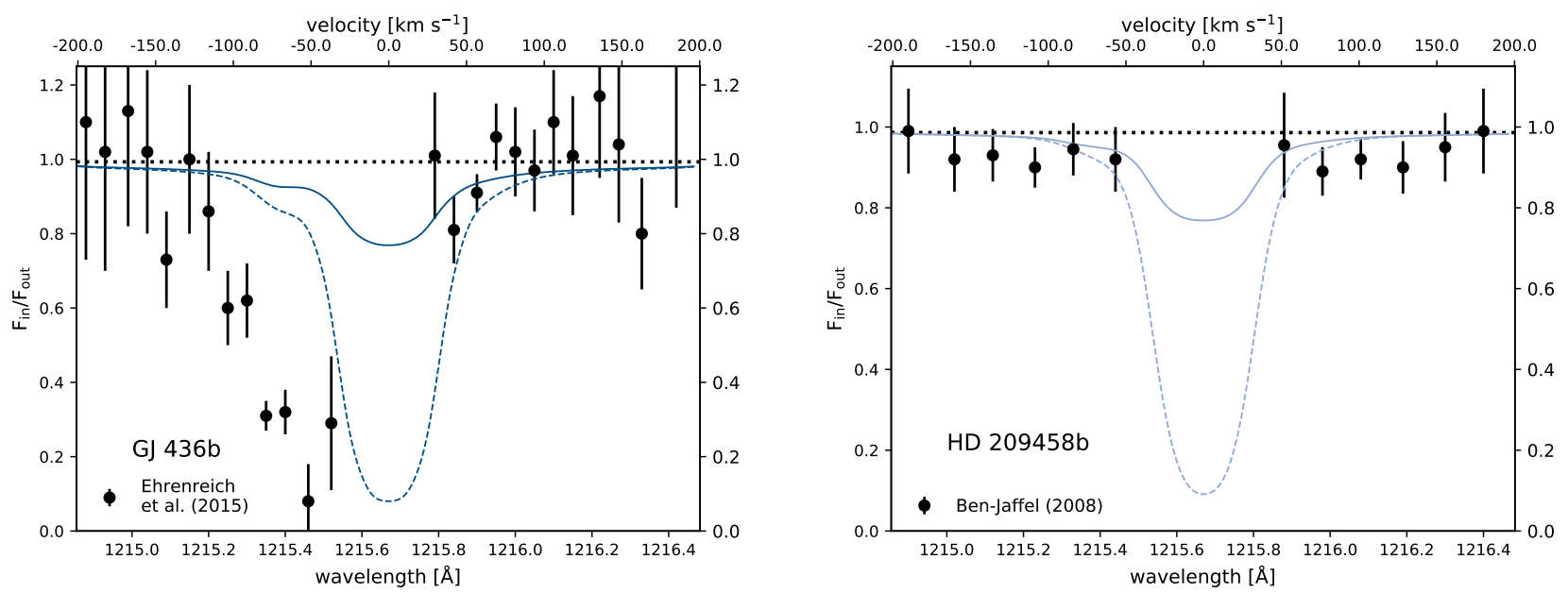

Figure 5. Ly $\alpha$ transmission spectra for planets with properties like GJ 436b and HD 209458b, transiting across the center of the stellar disk. Solid lines show the absorption caused by hydrogen located within the Roche radius, whereas the dashed lines take into account gas at all altitudes. Transit observations are shown for comparison. The spectral region near the line core that is affected by the interstellar absorption and geocoronal emission is omitted from the observational data.

deuterium, assuming a deuterium fraction of $2.25 \times 10^{-5}$, as measured in Jupiter by Lellouch (2001)). We calculate the hydrogen Ly $\alpha$ line profiles using the wavelengths, oscillator strengths, and natural broadening parameters from the NIST database.

In Figure 5 we show the obtained line profiles for both planets (convolved with the HST/STIS line-spread function for G140M grating and aperture of $\left.52^{\prime \prime} \times 0^{\prime \prime} .1\right)$ and compare them with observations from Ben-Jaffel (2008) and Ehrenreich et al. (2015). Our simple wind model cannot fully explain the observed absorption in the wings of the Ly $\alpha$ line due to the simplifications discussed in section 4. The Ly $\alpha$ line center - where our model should be more reliable -is observationally unattainable due to the interstellar absorption and geocoronal emission. Our results agree reasonably well with the results of theoretical studies by Ben-Jaffel \& Sona Hosseini (2010) and Salz et al. (2016).

\section{REFERENCES}

Andretta, V. \& Jones, H. P. 1997, ApJ, 489, 375

Avrett, E. H., Fontenla, J. M., Loeser, R. 1994, in IAU

Symposium, Vol. 154, Infrared Solar Physics, ed. D. M.

Rabin, J. T. Jefferies, C. Lindsey, 35

Ben-Jaffel, L. 2008, ApJ, 688, 1352

Ben-Jaffel, L. \& Sona Hosseini, S. 2010, ApJ, 709, 1284

Bourrier, V., \& Lecavelier des Etangs, A. 2013, A\&A, 557, A124

Bourrier, V., Lecavelier des Etangs, A., Ehrenreich, D.,

Tanaka, Y. A., \& Vidotto, A. A. 2016, A\&A, 591, A121

Bray, I., Burgess, A., Fursa, D. V., \& Tully, J. A. 2000,

A\&AS, 146, 481

Brown, R. L. 1971, ApJ, 164, 387

Butler, R. P., Vogt, S. S., Marcy, G. W., et al. 2004, ApJ, 617,580

Carroll-Nellenback, J., Frank, A., Liu, B., et al. 2017, MNRAS, 466, 2458

Charbonneau, D., Brown, T. M., Latham, D. W., \& Mayor, M. 2000, ApJL, 529, L45
Christie, D., Arras, P., \& Li, Z.-Y. 2016, ApJ, 820, 3

Czesla, S., Salz, M., Schneider, P. C., Mittag, M., Schmitt, J. H. M. M. 2017, A\&A, 607, A101

Drake, G. W. 1971, PhRvA, 3, 908

Dupree, A. K., Sasselov, D. D., \& Lester, J. B. 1992, ApJL, $387, \mathrm{~L} 85$

Edwards, S., Fischer, W., Kwan, J., Hillenbrand, L., \& Dupree, A. K. 2003, ApJL, 599, L41

Ehrenreich, D., Bourrier, V., Wheatley, P. J., et al. 2015, Nature, 522, 459

France, K., Parke Loyd, R. O., Youngblood, A., et al. 2016, ApJ, 820, 89

Fossati, L., Haswell, C. A., Froning, C. S. et al. 2010, ApJL, 714, L222

Fulton, B. J., Petigura, E. A., Howard, A. W. et al. 2017, AJ, 154, 109

García Muñoz, A. 2007, Planet. Space Sci., 55, 1426

Henry, G. W., Marcy, G. W., Butler, R. P., \& Vogt, S. S. 2000, ApJL, 529, L41 
Hill, C. 2016, Learning Scientific Programming with Python (Cambridge: Cambridge Univ. Press)

Hu, R., Seager, S. and Yung, Y. L. 2015, ApJ, 807, 8

Hunter, J. D., et al. 2007, Computing in science and engineering, 9, 90

Indriolo, N., Hobbs, L. M., Hinkle, K. H. and McCall, B. J. 2009, ApJ, 703, 2131

Jones, E., Oliphant, T., Peterson, P., et al. 2001, SciPy: Open source scientific tools for Python

Khodachenko, M. L., Shaikhislamov, I. F., Lammer, H., Prokopov, P. A. 2015, ApJ, 813, 50

Koskinen, T. T., Yelle, R. V., Lavvas, P., \& Lewis, N. K. 2010, ApJ, 723, 116

Kulow, J. R., France, K., Linsky, J., \& Loyd, R. O. P. 2014, ApJ, 786, 132

Lamers, H. J. G. L. M., \& Cassinelli, J. P. 1999, Introduction to Stellar Winds, 452

Lammer, H., Selsis, F., Ribas, I., et al. 2003, ApJL, 598, L121

Lavie, B., Ehrenreich, D., Bourrier, V., Lecavelier des Etangs, A., Vidal-Madjar, A., Delfosse, X., Gracia Berna, A., Heng, K.,Thomas, N., Udry, S., Wheatley, P. J. 2017, A\&A, 605, L7

Lecavelier Des Etangs, A., Ehrenreich, D., Vidal-Madjar, A., et al. 2010, A\&A, 514, A72

Leighly, K. M., Dietrich, M., \& Barber, S. 2011, ApJ, 728, 94

Lellouch, E., Bézard, B., Fouchet, T. et al. 2001, A\&A, 370, 610

Linsky, J. L., Yang, H., France, K., et al. 2010, ApJ, 717, 1291

Linsky, J. L., Fontenla, J., France, K. 2014, ApJ, 780, 61

Loyd, R. O. P., France, K., Youngblood, A., et al. 2016, ApJ, 824, 102

Lunkdkvist, M. S., Kjeldsen, H., Albrecht, S. et al. 2016, Nature Communications, 7, 11201

Mauas, P. J. D., Andretta, V., Falchi, A., Falciani, R., Teriaca, L., Cauzzi, G. 2005, ApJ, 619, 604
Moutou, C., Coustenis, A., Schneider, J., Queloz, D., \& Mayor, M. 2003, A\&A, 405, 341

Murray-Clay, R. A., Chiang, E. I., \& Murray, N. 2009, ApJ, 693,23

Norcross, D. W. 1971, Journal of Physics B Atomic Molecular Physics, 4, 652

Osterbrock, D. E., \& Ferland, G. J. 2006, Astrophysics of gaseous nebulae and active galactic nuclei

Owen, J. E., \& Jackson, A. P. 2012, MNRAS, 425, 2931

Owen, J. E., \& Wu, Y. 2012, ApJ, 775, 105

Parker, E. N. 1958, ApJ, 128, 664

Roberge, W., \& Dalgarno, A. 1982, ApJ, 255, 489

Salz, M., Banerjee, R., Mignone, A., et al. 2015, A\&A, 576, A21

Salz, M., Czesla, S., Schneider, P. C., \& Schmitt, J. H. M. M. 2016, A\&A, 586, A75

Schneiter, E. M., Esquivel, A., DAngelo, C. S. V., et al. 2016, MNRAS, 457, 1666

Seager, S., \& Sasselov, D. D. 2000, ApJ, 537, 916

Tripathi, A., Kratter, K. M., Murray-Clay, R. A., \& Krumholz, M. R. 2015, ApJ, 808, 173

Turner, J. D., Christie, D., Arras, P., Johnson, R. E., \& Schmidt, C. 2016, MNRAS, 458, 3880

Van Der Walt, S., Colbert, S. C., \& Varoquaux, G. 2011, Computing in Science \& Engineering, 13, 22

Vidal-Madjar, A., Lecavelier des Etangs, A., Dsert, J.-M., et al. 2003, Nature, 422, 143

Vidal-Madjar, A., Dsert, J.-M., Lecavelier des Etangs, A., et al. 2004, ApJL, 604, L69

Villarreal D'Angelo, C., Schneiter, M., Costa, A., et al. 2014, MNRAS, 438, 1654

Watson, A. J., Donahue, T. M., Walker, J. C. G. 1981, Icarus, 48, 150

Yelle, R. V. 2004, Icarus, 170, 167

Youngblood, A., France, K., Parke Loyd, R. O., et al. 2016, ApJ, 824, 101 